\title{
Praktische Erfahrungen mit dem Rambach-Agar
}

\section{Experiences in the use of Rambach Agar}

H. E. Müller

Staatliches Medizinaluntersuchungsamt Braunschweig

\section{Zusammenfassung:}

Der Rambach-Agar enthält mit Propylenglykol und dem Farbstoff 5-Brom-4chlor-3-indolyl-ß-D-galactopyranosid zwei Substanzen, die in keinem anderen Medium fürdie Salmonellendifferenzierung enthalten sind. Das Originalrezept von Rambach wurde in folgenden Punkten modifiziert: Statt 0,5\% Pepton und 0,2\% Hefeextrakt wurden 0,7\% Hefeextrakt verwendet und der pH-Wert auf 6, 1 eingestellt. Dadurch wird der Anteil der charakteristisch als rote und nicht mehr als helle Kolonien wachsenden Salmonellen erhöht. Die Sensitivität und Spezifität des Rambach-Agar wurden im Direktausstrich von Stuhlproben verdächtiger Patienten mit Endo-, SS-, Wilson-Blair- und XLDAgar verglichen. In beiden Untersuchungsansätzen war der XLD-Agar das beste Medium, der Rambach-Agar das zweitbeste und besser als der WilsonBlair-Agar. Auch Endo- und SS-Agar waren im Direktausstrich weniger leistungsfähig als der Rambach-Agar. Deshalb erscheint die Kombination von XLD- und Rambach-Agarals die beste Kombination von zwei SalmonellaDifferentialnährböden.

\section{Schlüsselwörter:}

Salmonellendiagnostik - Rambach-Agar - Sensitivität - Spezifität

\section{Summary:}

The Rambach agar consists of propylene glycol and the chromogen 5bromo-4-chloro-3-indolyl- $B$-D-galactopyranoside. There are no other media for differentiation of salmonellae containing these substances. The composition, originally described by Rambach, was improved by two modifications: $0.7 \%$ yeast extract were used instead of $0.5 \%$ peptone and $0.2 \%$ yeast extract. In addition, more Salmonella colonies show a bright red color by adjustment of the $\mathrm{pH}$ to 6.1. After direct plating of stool samples, the sensitivity and specificity of Rambach agar was compared with Endo, SS, Wilson Blair, and $X L D$ agar. Furthermore, the sensitivity and specificity of Rambach agar were compared with that of Wilson Blair and XLD agar after enrichment of stool samples in Cystin selenite and Preuss'tetrathionate broth. Both investigations showed that the XLD agar was the best followed by the Rambach agar whereas the Wilson Blair agar and also Endo and SS agar were less effective than the Rambach agar. Therefore, the combination of XLD and Rambach agar seems to be an optimum combination of two media for differentiation of salmonellae.

\section{Keywords:}

Diagnosis of salmonella - Rambach agar - sensitivity - specificity

\section{Einleitung}

1990 beschrieb Rambach ein neues Salmonellenmedium (12). Zur Selektierung dient das auch in vielen anderen Nährböden verwendete Natriumdesoxycholat. Zur Differenzierung wurden zwei Substanzen eingeführt, die bisher in keinem anderen Salmonellenagar enthalten waren: Alle Bakterien, die ß-Galaktosidase besitzen und Laktose spalten, können aus dem Farbstoff 5-Brom-4-chlor3-indolyl-B-D-galactopyranosid einen blauen Farbstoff freisetzen. Dadurch entstehen blaue, blau-grüne oder violett gefärbte Kolonien. Der Nachweis der Salmonellen geschieht mit Propylenglykol. Bei seiner Verwertung kommt es zur Säuerung des Nährbodens und die Salmonellenkolonien färben sich mit Neutralrot leuchtend karmesinrot an (2). Nur S. typhi und wenige Stämme anderer Salmonella-Serovare, die insgesamt weniger als $2 \%$ der anfallenden Isolate ausmachen, wachsen ähnlich wie Acinetobacter, Alcaligenes, Morganella, Proteus, Providencia, Shigella und Vibrio-Arten als helle Kolonien (3). 


\section{Originalie}

Die bisherigen Erfahrungsberichte kamen zu recht unterschiedlichen Bewertungen. Einerseits wurde die Sensitivität des Rambach-Agars mit $90 \%$ (6), aber andererseits auch nur mit 59-68\% (4) bzw. mit 69\% (1) beschrieben. Da zudem die angebotenen Fertigmedien für den Routinebetrieb ziemlich kostspielig und die notwendigen Ingredienzien relativ preiswert sind, wurde der Nährboden in der eigenen Nährbodenküche hergestellt, dabei seine Sensitivität und Spezifität optimiert und mit zwei Nährböden verglichen, die sich in früheren Untersuchungen als die damals besten erwiesen hatten $(7,8,13)$.

\section{Material und Methoden}

\section{Procedere der Untersuchung}

Die Untersuchungen zum Vergleich des Rambach-Agars erfolgten exakt nach dem gleichen Procedere,das auch bei der routinemäßigen Stuhluntersuchung auf Salmonellen durchgeführt wird:

1) Die Stuhlproben wurden zunächst direkt auf Endo-, SS- (= Salmonella-Shigella-Agar), Wilson-Blair-Agar (= Bismut-Sulfit-Agar), XLD-Agar (= Xylose-Lysin-Desoxycholat-Agar) und vergleichend auf Rambach-Agar ausgestrichen und nach eintägiger Bebrütung bei $36 \pm 1^{\circ} \mathrm{C}$ beurteilt.

2) Außerdem wurden die Stuhlproben parallel in SelenitCystin- und in Tetrathionat-Brühe nach Preuss über Nacht bei $36 \pm 1^{\circ} \mathrm{C}$ bebrütet, danach aus beiden Anreicherungsbrühen auf die drei Nährböden ausgestrichen, und nach ein- und zweitägiger Bebrütung bei $36 \pm 1^{\circ} \mathrm{C}$ abgelesen.

\section{Kulturmedien}

Verschiedene Peptone (Lab Lemco, Peptone Special, Proteose Peptone und Tryptose) Yeast Extract, SS- und
XLD-Agar wurden von Oxoid, Wesel bezogen, Reinsubstanzen von E. Merck, Darmstadt, Propylenglykol von Sigma, Deisenhofen/München und der Farbstoff 5-Brom4-chlor-3-idolyl-ß-D-galactopyranosid von Biosynth, Staad/Schweiz. Der Endo- und der Wilson-Blair-Agar sowie die Selenit-Cystin- und die Tetrathionat-Brühe nach Preuss wurden aus den Ingredienzien in der eigenen Nährbodenküche nach andernorts beschriebenen $\mathrm{Re}$ zepten hergestellt $(8,9,10, \mathbf{i 1})$.

\section{Bakterien}

Die Differenzierung der Salmonellen erfolgte biochemisch, ferner mit Hilfe des 01-Phagen sowie serologisch nach üblichen Verfahren. Ebenso wurden auch die anderen Bakterien der Stuhlflora bestimmt, soweit das für die Diagnostik notwendig war (5).

\section{Ergebnisse und Diskussion}

Die Pepton- und Desoxycholat-Konzentration des Rambach-Agar ist so gewählt, daß die meisten Bakterienarten, die weder das Propylenglykol nach die Galaktose aus 5-Brom-4-chlor-3-idolyl- $ß-D$-galactopyranosid verwerten, nicht nur als helle, sondern auch als relativ kleine Kolonien wachsen. Allerdings war bei unseren Untersuchungen eine Konzentration von $0,7 \%$ Hefeextrakt günstiger als die von Rambach vorgeschlagene Kombination von $0,2 \%$ Hefeextrakt und $0,5 \%$ Pepton. Ferner machte Rambach in seiner Originalpublikation (12) keine Angaben zum pH-Wert des Mediums. Die Hersteller von gebrauchsfertigen Rambach-Agar-Platten wählten pH-Werte von 7,4 wie etwa Technogram, Paris, oder 7,3 wie $E$. Merck, Darmstadt. Nach - unseren Erfahrungen erwies sich jedoch ein auf $\mathrm{pH} 6,1$ abgesenkter Wert als günstiger, weil dadurch viele Stämme der sonst hell oder nur schwach rosa gefärbten $S$. enteritidis-Kolonien eine leuchtend rote Farbe annahmen.

Tab. 1: Vergleich von Rambach-Agar mit Endo-, SS-, Wilson-Blair- und XLD-Agar nach Direktausstrich sowie im Vergleich mit Wilson-Blairund XLD-Agar nach Anreicherung in Selenit-Cystin- und Tetrathionat-Brühe nach Preuss. Sensitivität als prozentuale Verhältniszahl der positiven Salmonellen-Befunde auf dem jeweiligen Nährboden zur Gesamtzahl der positiven einschließlich der falsch negativen Befunde und Spezifität als prozentuale Verhältniszahl der negativen Salmonellen-Befunde auf dem jeweiligen Nährboden zur Gesamtzahl der negativen einschließlich der falsch positiven Befunde.

\begin{tabular}{|c|c|c|c|c|c|c|c|c|c|}
\hline $\begin{array}{l}\text { Ant der } \\
\text { Untersuchung }\end{array}$ & Nährboden & $\begin{array}{l}\text { Gẹsamtzahl } \\
\text { der Unter- } \\
\text { suchungen }\end{array}$ & $\begin{array}{l}\text { insgesamt } \\
\text { positiv }\end{array}$ & $\begin{array}{l}\text { auf dem Nährboden } \\
\text { positiv falsch } \\
\text { negativ }\end{array}$ & $\begin{array}{l}\text { Sensiti- } \\
\text { vität } \\
\text { (\%) }\end{array}$ & $\begin{array}{l}\text { Insgesamt } \\
\text { negativ }\end{array}$ & $\begin{array}{l}\text { auf dem Nāhrboden } \\
\text { negativ falsch } \\
\text { positiv }\end{array}$ & $\begin{array}{l}\text { Spezi- } \\
\text { fität } \\
(\%)\end{array}$ & $\begin{array}{l}\text { Summe von } \\
\text { Sensitivität + } \\
\text { Spezilität (\%) }\end{array}$ \\
\hline
\end{tabular}

\begin{tabular}{|c|c|c|c|c|c|c|c|c|c|c|c|c|}
\hline \multirow[t]{5}{*}{ Direktausstrich } & Rambach-Agar & 5456 & 438 & 151 & 287 & $34,5 \%$ & & 5018 & 5161 & 143 & $97,2 \%$ & $131,7 \%$ \\
\hline & Endo-Agar & 5456 & 438 & 165 & 273 & $37,7 \%$ & & 5018 & 6235 & 1217 & $80,5 \%$ & $118,2 \%$ \\
\hline & SS-Agar & 5456 & 438 & 142 & 296 & $32,4 \%$ & & 5018 & 5841 & 823 & $85,9 \%$ & $118,3 \%$ \\
\hline & Wilson-Blair-Agar & 5456 & 438 & 136 & 302 & $31,1 \%$ & $\therefore$ & 5018 & 5971 & 953 & $84,0 \%$ & $115,1 \%$ \\
\hline & XLD-Agar & 1586 & 114 & 48 & 66 & $42,1 \%$ & - & 1472 & 498 & 26 & $98,3 \%$ & $140,4 \%$ \\
\hline Ausstrich nach & Rambach-Agar & 7498 & .1531 & 1288 & 243 & $84,1 \%$ & & 5967 & 5122 & 845 & $85,8 \%$ & $169,9 \%$ \\
\hline \multirow[t]{2}{*}{ Anreicherung } & Wilson-Blair-Agar & 7498 & 1531 & 807 & 724 & $52,7 \%$ & & 5967 & 5918 & 49 & $99,2 \%$ & $151,9 \%$ \\
\hline & XLD-Agar & 7498 & 1531 & 1384 & 147 & $90,4 \%$ & & 5967 & 4831 & 1136 & $81,0 \%$ & $171,4 \%$ \\
\hline
\end{tabular}


Nach wie vör hell wächst allerdings S. typhi. Das ist zweifelsohne das größe Handikap des Rambach-Agars. Immerhin ist Typhus schon heute eine Importkrankheit, die in Deutschland praktisch nicht mehr vorkommt. $\mathrm{Zu}$ dem wird man Typhuserreger primär aus der Blutkultur züchten. Dagegen erscheinen sie im Stuhl erst so spät, daß eine positive Stuhlprobe nur noch geringe therapeutische Relevanz besitzt.

Die Leistungsfähigkeit des Rambach-Agars im Vergleich mit einigen anderen Selektivmedien ist in Tab. 1 dargestellt. Als Maßzahlen sind die Sensitivität und Spezifität des Rambach-Agars im Vergleich mit Endo-, SS-. WilsonBlair- und XLD-Agar beim Direktausstrich von Stuhlproben und ferner im Vergleich mit Wilson-Blair- und XLDAgar nach vorheriger Anreicherung in Selenit-Cystinund Tetrathionat-Brühe nach Preuss angegeben. Daraus läßt sich ablesen, daß der Rambach-Agar nach dem XLDAgar sowohl bei Direktausstrich der Stuhlproben als auch bei Ausstreichen der in Selenit-Cystin- und Tetrathionat-Brühen angereicherten Stuhlproben die zweitbesten Resultate liefert und deutlich besser ist als der in unserem Labor bisher benutzte Wilson-Blair-Agar. In beiden Versuchsserien besitzt XLD-Agar die höchste und der Wilson-Blair-Agar die niedrigste Summe aus Sensitivität + Spezifität.

Im vorliegenden Fall schnitt der XLD-Agar besser ab als der Rambach-Agar, in der Untersuchung von Dusch und Altwegg war es der Hektoen-Agar. Auch diese Autoren fanden bei ihren Vergleichsuntersuchungen mit zwei anderen Selektivnährboden, daß der Rambach-Agar eine mittlere Position einnimmt (1). Dagegen isolierte Heizmann bei Direktausstrichen von zwei parallel angelegten SS-Agarplatten mehr Salmonellen als von einer einzigen Rambach-Agarplatte (4). Allerdings zeigt Tab. 1, daß vom Rambach-Agar unter vergleichbaren Bedingungen mehr Salmonellen isoliert werden können als von SS-Agar.

Bei allen diesen Zahlen muß zusätzlich berücksichtigt werden, daß die Beurteilung eines neuen Nährbodens im Vergleich mit den bisherbenützten und vertrauten Medien notwendigenweise zu schlecht ausfällt. Nach unseren Erfahrungen ist die Vertrautheit erst nach etwa drei Monaten so groß, daß das Ablesen der Nährböden zu vergleichbaren Ergebnissen führt. Die Validität von Aussagen über die Leistungsfähigkeit des Rambach-Agar im Vergleich mit anderen Differentialnährböden sind also ganz wesentlich von der Zahl der untersuchten Proben abhängig. Die relativ negative Beurteilung, die der Rambach-Agar in zwei anwendungsorientierten Studien erfuhr $(1,4)$, dürfte nicht zuletzt durch die insgesamt geringen Untersuchungszahlen bedingt sein. In engem $\mathrm{Zu}$ sammenhang damit steht der relativ hohe Preis der kommerziell vertriebenen Fertignährböden, der einem breiten Einsatz des Rambach-Agars im Wege steht, zumal die Leistungsfähigkeit des neuen Mediums zwar gut, aber sicher nicht besser ist als andere optimale Salmonella-Differentialnährböden. Aber Laboratorien mit eigener Nährbodenküche können sich den Rambach-Agar leicht selbst herstellen, denn die Ausgangssubstanzen sind verhältnismäßig preiswert.
Literatur:

1. Dusch, H. and Altwegg, M. (1993): Comparison of Rambach agar, SM-ID medium, and hektoen enteric agar for primary isolation of non-typhi salmonellae from stool samples. Journal of Clinical Microbiology 31, 410-412.

2. Freydiere, A.-M. and Gille, Y. (1991): Detection of salmonellae by using Rambach agar and by a C8 esterase spot test. Journal of Clinical Microbiology 29, 2357-2359.

3. Gruenewald, R.; Henderson, R.W. and Yappow, S. (1991): Use of Rambach propylene glycol containing agar for identification of Salmonella spp. Journal of Clinical Microbiology 29, 2354-2356.

4. Heizmann, W.R. (1993) Detection of Salmonella in human faeces by Rambach agar and by a color latex agglutination assay. Medical Microbiology Letters 2, 131-137.

5. Holt, J.G.; Krieg, N.R.; Sneath, P.H.A.; Staley, J.T. and Williams, S.T (1994): Bergey's Manual of Determinative Bacteriology. 9th Ed., Williams \& Wilkins, Baltimore, Maryland.

6.Manafi, M. and Sommer, R. (1992): Comparison of three rapid screening methods for Salmonella spp.: 'MUCAP test, MicroScreenR Latex and Rambach agar. Letters in Applied Microbiology 14, 163-166.

7. Metz, H.; Müller, H.E. (1981): Ergebnisse einer Umfrage bei 84 Laboratorien über ihre Salmoneila- und Shigella-Diagnostik im Jahr 1979. Bundesgesundheitsblatt 24, 315-324.

8. Müller, H.E. (1980): Vergleichende Untersuchungen über die Effizienz von Selenit- und Tetrathionat-Brühe und von Leifson- und Wilson-Blair-Agar bei der Isoliergung von Salmonellen. Zentralblatt für Bakteriologie Mikrobiologie und Hygiene I. Abt. Originale A 248, 202-209.

9. Müller, H.E. (1982): Ein Methodenvergleich zur Anreicherung von Salmonellen aus menschlichen Faeces. Zentralblatt für Bakteriologie Mikrobiologie und Hygiene l. Abt. Originale A 253, 183-196.

10. Müller, H.E. (1991): Verschiedene Möglichkeiten der SalmonellenAnreicherung unter besonderer Berücksichtigung der nach DAB 9 empfohlenen Methode. Die Pharmazeutische Industrie 53, 677-681.

11. Müller, H.E. (1992): Eine Funktionsanalyse der Komponenten der Tetrathionat-Bouilion nach Preuss zur Anreicherung von Salmonellen. Laboratoriumsmedizin 16, 149-155.

12. Rambach, A. (1990): New plate medium for facilitated differentiation of Salmonella spp. from Proteus spp. and other enteric bacteria. Applied and Environmental Microbiology 56, 301-303.

13. Schomburg, I.; Näveke, R.; Müller, H.E. (1983): Das Wachstum von Salmonellen auf verschiedenen Selektivagarmedien. Laboratoriumsmedizin 7, $W+F$ 172-176.

\section{Danksagung:}

Für die gewissenhafte Durchführung der Untersuchungen danke ich Frau Friederike Heimbach und Frau Sabine Warmbold.

Anschrift des Verfassers:

Prof. Dr. med., Dr. rer. nat. Hans E. Müller

Staatliches Medizinaluntersuchungsamt Braunschweig

Hallestraße 1

38124 Braunschweig 


\section{Hinweise für Autoren zur Abfassung von Manuskripten}

Die Zeitschrift LABORATORIUMSMEDIZIN erscheint $11 \mathrm{mal}$ jährlich, für die Monate Juli/August als Doppelheft.

Veröffentlicht werden Originalarbeiten, Übersichtsreferate und Kurzmitteilungen auf dem Gebiet der Laboratoriumsmedizin (Klinische Chemie, Bakteriologie-Serologie, Immunologie, Hämatologie. Hämostaseologie, Zytologie, Zytogenetik) in deutscher und englischer Sprache.

Manuskript in Schriftform und wenn möglich auf Diskette einsenden.

\section{Annahmebedingungen für Originalarbeiten}

1. Die wissenschaftlichen Ergebnisse dürfen noch nicht veröffentlicht sein, die Autoren müssen das Urheberrecht besitzen.

2. Umfang des Manuskriptes bis zu 20 Schreibmaschinenseiten inklusive Literatur, Abbildungen und Tabellen.

3. Gliederung in:

Titel (deutsch und englisch),

Zusammenfassung (deutsch und englisch),

Schlüsselwörter (deutsch und englisch),

Einleitung, Material und Methoden, Ergebnisse, Diskussion, Literatur.

Der Ergebnisteil muß auch die Interpretation der Ergebnisse beinhalten. In der Diskussion sollen die Ergebnisse kritisch zu den Arbeiten anderer Arbeitsgruppen gleicher Forschungsrichtung beurteilt werden, ferner soll ein Bezug zur klinischen Anwendung hergestellt werden.

4. Arbeiten, die einen direkten Beitrag zu aktuellen Fragestellungen der Laboratoriumsmedizin liefern, werden bevorzugt angenommen und schneller veröffentlicht.

\section{Annahmebedingungen für Übersichtsreferate}

1. Das Thema muß aktuell sein und außer Ärzte für Laboratoriumsmedizin und Naturwissenschaftler im medizinischen Labor mindestens eine weitere Gruppe von Fachärzten und Allgemeinärzten ansprechen.

2. Umfang des Manuskriptes bis zu 20 Schreibmaschinenseiten inklusive Literatur, Abbildungen und Tabellen.

3. Die Gestaltung ist frei, zwingend sind jedoch: Titel (deutsch und englisch), Zusammenfassung (deutsch und englisch), Schlüsselwörter (deutsch und englisch) sowie die Gliederung in Einleitung, Methoden, Ergebnisse, Diskussion und Literatur.

\section{Annahmebedingungen für Kurzreferate}

1. Vorwiegend methodische oder klinisch-diagnostische Arbeiten sowie Erfahrungen oder Hinweise zur Praxis oder Fortbildung auf dem Gebiete der Laboratoriumsmedizin werden angenommen.

2. Umfang des Manuskriptes bis zu 4 Schreibmaschinenseiten.

3. Gestaltung wie Originalarbeiten.

4. Veröffentlichung gewöhnlich innerhalb von 2 Monaten nach Annahmedatum.

\section{Gestaltung der Manuskripte}

1. Seite:Titel des Beitrages in deutsch und englisch, abgekürzter Vorname und Nachname der Autoren (bei Frauen ausgeschriebener Vorname), Klinik, Institut.

2. Seite: Zusammenfassung, Schlüsselwörter in deutsch und englisch.

Letzte Seite: Literaturverzeichnis und Autorenanschriften.

\section{Literaturzitation:}

Die Literaturzitate werden in folgender Reihenfolge numeriert, also nicht alphabetisch. Die Literaturstellen werden durch Name, Vorname, Erscheinungsjahr, Titel der Arbeit, Zeitschriftenname, Band-Nummer und Seite in dieser Reihenfolge gekennzeichnet.

Beispiel Originalarbeit: Thomas, L. (1990): Quantitative immunchemische Plasmaproteinbestimmung mittels Nephelometrie und Turbidimetrie. Lab.med. 14, 313-320.

Beispiel Bücher: Felgenhauer, K. (1992) Liquordiagnostik. In: Labor und Diagnose - Indikation und Bewertung von Laborbefunden für die medizinische Diagnostik (Thomas, L., ed.). Die Medizinische Verlagsgesellschaft Marburg, Germany. pp. 1715-1740.

Am Ende des Manuskriptes sollen aufgeführt werden: Ausgeschriebene Vor- und Nachnamen sowie Anschriften aller Autoren, und zwar zuerst der Name jenes Autors, bei dem Sonderdrucke angefordert werden können.

Abbildungen, Tabellen und Diagramme: Die Abbildungen sollen reproduktionsreif sein. Für Originale übernimmt der Verlag keine Haftung. Symbole, Einheiten, Buchstaben und Zahlen müssen in Rundschrift und so groß gezeichnet werden, daß sie auch nach der Verkleinerung gut lesbar sind.

Die Legenden zu den Abbildungen und Tabellen sollen auf einem gesonderten Blatt sein. Abbildungen müssen auf der Rückseite die Nummer der Abbildung, den Namen des ersten Autors und die gewünschte Position im Text tragen.

Anzahl der einzusendenden Manuskripte: 3 Ausdrucke.

Schreibweise: maßgebend ist der Duden.

\section{Begutachtung}

Der verantwortliche Schriftleiter entscheidet über die Annahme nach Einholung von 2 sachverständigen Gutachten. Die Gutachter bleiben gegenüber den Autoren anonym und beurteilen unabhängig. Die Autoren erhalten eine Kopie der Gutachten und spätestens 2 Monate nach Eingang des Manuskriptes die Entscheidung über Annahme oder Ablehnung sowie einen vorläufigen Termin der Veröffentlichung.

\section{Korrekturen}

Dem federführenden Autor werden die Umbruchseiten zur Korrektur übersandt. Er hat dafür Sorge zu tragen, daß auch im Falle seiner Abwesenheit die korrigierten Seiten innerhalb von maximal 2 Wochen dem Verlag zurückgesandt werden. In den Druckfahnen soll nur in Ausnahmefällen und bei eindeutigen Fehlern, deren Korrektur übersehen wurde, berichtigt werden. Deshalb darf jedes Manuskript erst nach sorgfältiger Prüfung aller Gesichtspunkte zur endgültigen Fassung der Schriftleitung eingereicht werden. Nachträgliche Textänderungen dürfen 2 Prozent des Textes nicht überschreiten.

\section{Veröffentlichung}

Zielsetzung ist es, jede Arbeit innerhalb von 6 Monaten nach Eingang $\mathrm{zu}$ veröffentlichen. Der einsendende Autor erhält nach Veröffentlichung ein Belegheft sowie 50 Sonderdrucke.

Einsendung: Original und 2 Kopien, wenn möglich auch Diskette, an:

Schriftleitung Wissenschaft und Fortbildung der Zeitschrift LABORATORIUMSMEDIZIN

Prof. Dr. L. Thomas, Kirschbaumweg 8, D-60489 Frankfurt 